Article

\title{
Design Optimization of an Electric Variable Transmission for Hybrid Electric Vehicles
}

\author{
Qiwei Xu ${ }^{1, *}$, Jing Sun ${ }^{1}$, Wenjuan Wang ${ }^{1}$, Yunqi Mao ${ }^{1}$ and Shumei Cui ${ }^{2}$ \\ 1 State Key Laboratory of Power Transmission Equipment \& System Security and New Technology, \\ Chongqing University, Chongqing 400044, China; sunjingzzx@cqu.edu.cn (J.S.); \\ irenewangwj@gmail.com (W.W.); myq2016@cqu.edu.cn (Y.M.) \\ 2 Department of Electrical Engineering, Harbin Institute of Technology, Harbin 150080, China; \\ cuism@hit.edu.cn \\ * Correspondence: xuqw@cqu.edu.cn; Tel./Fax: +86-23-6510-2434
}

Received: 20 April 2018; Accepted: 30 April 2018; Published: 2 May 2018

check for updates

\begin{abstract}
An electric variable transmission (EVT) for hybrid electric vehicles (HEVs) is investigated in this paper. With a special double rotor structure, the EVT splits and reintegrates the output power of the internal combustion engine (ICE) to run at its optimum working efficiency. However, the high electromagnetic coupling degree causes torque ripple and affects the dynamic performance of the EVT. After introducing the configuration and working principle, the torque mathematical model of the EVT in an ABC three-phase coordinate system is proposed to analyze the cause of this torque ripple. Besides, a finite element method-based (FEM) structural optimization design for reducing the torque ripple and improving the working stability is presented. The magnetic field distribution, induced voltage and torque property validate the rationality of optimization.
\end{abstract}

Keywords: hybrid electric vehicles; electric variable transmission; torque ripple; torque mathematical model; structural optimization design

\section{Introduction}

In recent years, with the rapid development of industry and the continuous increase of population, the "energy crisis" has become an urgent problem that needs to be solved immediately, therefore, new-energy vehicles came into being [1]. Battery-based electric vehicles (EVs), which have the benefits of zero direct emissions, non-pollution, high efficiency and energy-saving, are the future development direction of the automobile industry. Due to the low energy density, low power density of batteries, and the fact auxiliary charging facilities are not pervasive, the widely popularization of battery-based EVs is hampered at present. The research on hybrid electric vehicles (HEVs) is more mature than battery-based EV as they are cleaner than traditional vehicles, and have the advantages of high driving force, strong endurance, high fuel economies, and less demand for external facilities [2,3]. Therefore, the common view of new-energy vehicles is that the HEVs must be developed first, and one cannot jump directly from the ICE to zero emissions with battery-based EVs. The fuel economies of parallel-series HEVs are the best among all kinds of HEVs, which makes them more suitable for urban working conditions with frequent starting and stopping [4].

One of the powertrain system in parallel-series HEVs is the EVT system, which combines two electric machines [5]. The structure particularity of EVTs can make ICE running in its efficiency region. However, the two electric machines are not only mechanically coupled, but also interact with each other through the electromagnetic field. Compared with the traditional motors (a stator and a rotor), the electromagnetic coupling in EVT will increase the control difficulty. On the other hand, the magnetic coupling can also reduce the power factor, power density, and increase the electromagnetic 
loss, which is one of the difficult problems to be solved in any EVT system. At present, national and international scholars are mainly focused on the following aspects to research the magnetic field decoupling of EVT [6-11]: (1) studying the mathematical model of EVTs based on coordinate transformation. The expressions of flux linkage, voltage and electromagnetic torque are determined under a transformed coordinate system to identify the decoupling control algorithm and select the new state variables. The decoupling control effect is related to the accuracy of the parameters; (2) Changing the magnetic field distribution in EVTs to reduce the degree of mutual interference. A flux-insulation ring is installed in the middle of outer rotor, or a magnetic field modulation ring is arranged between the stator and rotor. The flux-insulation ring is made of some non-magnetic material, so the inner and outer magnetic circuits are isolated from each other. Then the two excitation sources of the EVT (the magnetic potential of the inner rotor and stator) are chained with the inner and outer squirrel cage separately to form two independent closed magnetic circuits. The magnetic field modulation ring adopts the magnetic block to change the air-gap magnetic conductance based on the flux-modulated effect. It produces a large number of harmonic components in the air-gap to modulate the magnetic field distribution and transfer electromagnetic energy; (3) Studying the effect of the iron core material and lamination method on the magnetic flux fluidity to reduce the degree of mutual interference between the inner and outer magnetic field of the EVT. The main factors to be considered when selecting the core material are the working flux density, magnetic permeability, mechanical strength, loss, working environment and material prices. Different core lamination methods will affect the value of air-gap magnetic flux density, and thus affect the working performance of the machine; (4) Studying the factors that influence the inductance, back EMF, flux linkage and torque parameters by using FEM. The magnetic field coupling makes the inductance and the output torque of EVT fluctuate seriously. The parameters can be selected according to the factors affecting the parameter variation to optimize the structural design of the EVT.

This paper studies the operation stability of EVTs used for HEVs. By introducing the configuration and working principle it can be known that the EVT based on the electromagnetic energy conversion rule can be split and the output power of ICE reintegrated to make it operate in the optimum efficiency area independent of the road conditions, which decreases the emissions and fuel consumption. Nevertheless, the high degree of electromagnetic coupling between the inner and outer magnetic field causes torque ripple, thus reducing the running stability of HEVs. Next, to analyze the reason for torque ripple, a torque mathematical model of an EVT in an ABC three-phase coordinate system is proposed. From the mathematical model it can be seen that the magnetic field oversaturation and the small magnetic co-energy result in the nonlinear variation of machine parameters, and the effect can be weakened by proper parameters. Based on the analysis results, the influence of the structural parameters and the excitation current on the torque ripple are analyzed using FEM. Finally, the magnetic field distribution, induced voltage and torque properties validate the rationality of the structural design optimization, which reduces the torque ripple of EVT to an acceptable range to guarantee the running stability of HEVs.

\section{Configuration and Working Principle}

\subsection{Configuration}

A sectional view of the EVT and structure of a HEV based on EVT are shown in Figure 1. The EVT has a stator, an outer rotor, together with an inner rotor, as shown in Figure 1a. The stator and inner rotor are installed the three-phase windings, which are linked with the two inverters to complete the energy transmission [12]. Two layers of squirrel-cage are placed on both sides of the outer rotor. The EVT can be seen as a combination of an inner machine (IM) and an outer machine (OM). The IM comprises the outer rotor and inner rotor, which connects to the ICE as well as final gear, and also be called electric machine1 (EM1). The OM comprises the stator and outer rotor, which is called electric machine1 (EM2) and have the same speed as HEVs. 


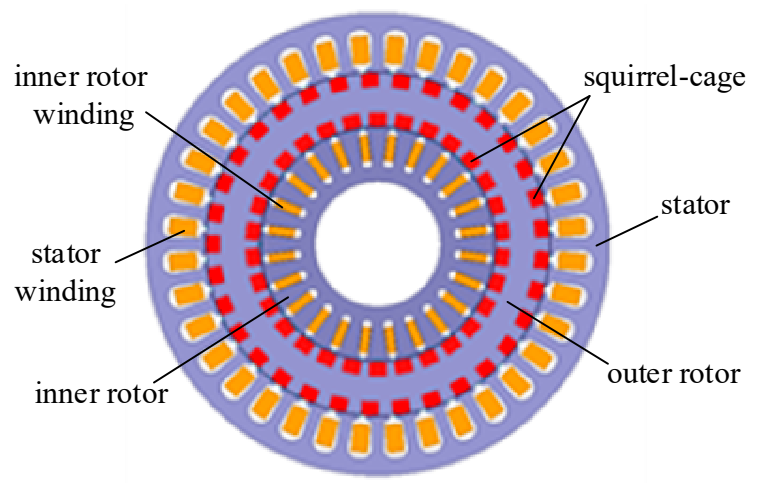

(a)

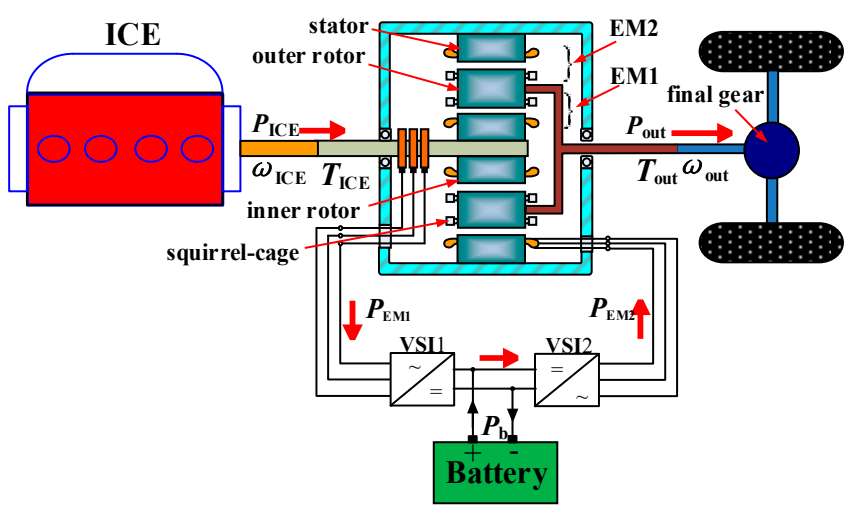

(b)

Figure 1. Structures of (a) sectional view of EVT and (b) HEV based on EVT.

The EVT is mounted between the ICE and the final gear, as shown in Figure 1b. The inner rotor is linked with ICE and the outer rotor is connected to the vehicle. To keep the ICE running in the efficiency region, the EM1 changes the speed of the outer rotor referring to the speed of ICE, and at the same time, transmits the torque of the ICE [13]. The EM2 provides the speed and torque difference between the ICE and vehicle, therefore, the speed and the torque of the ICE are adjusted simultaneously. The EVT combines the two machines together, making the transmission system of HEV more straightforward. However, the control strategies are complex, which need two individual conventional controllers, precise rotor position, as well as the proper control strategies. A 3D view of the EVT is shown in Figure 2.

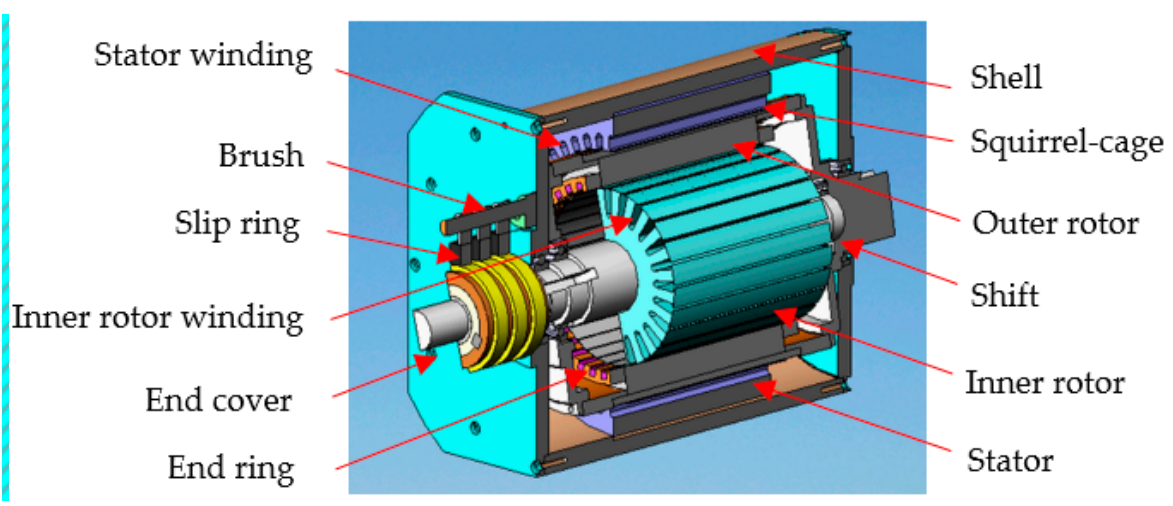

Figure 2. 3D view of the EVT. 


\subsection{Working Principle}

In a typical operation mode, the EVT alters the output speed and torque of the ICE by converting and transforming the mechanical energy of the ICE [14]. It achieves the splitting and reintegration of the output power of the ICE, which not only meets the requirements of the HEV driving force, but also optimizes the operation area of the ICE. The power split in the HEV based on EVT is shown in Figure 3.

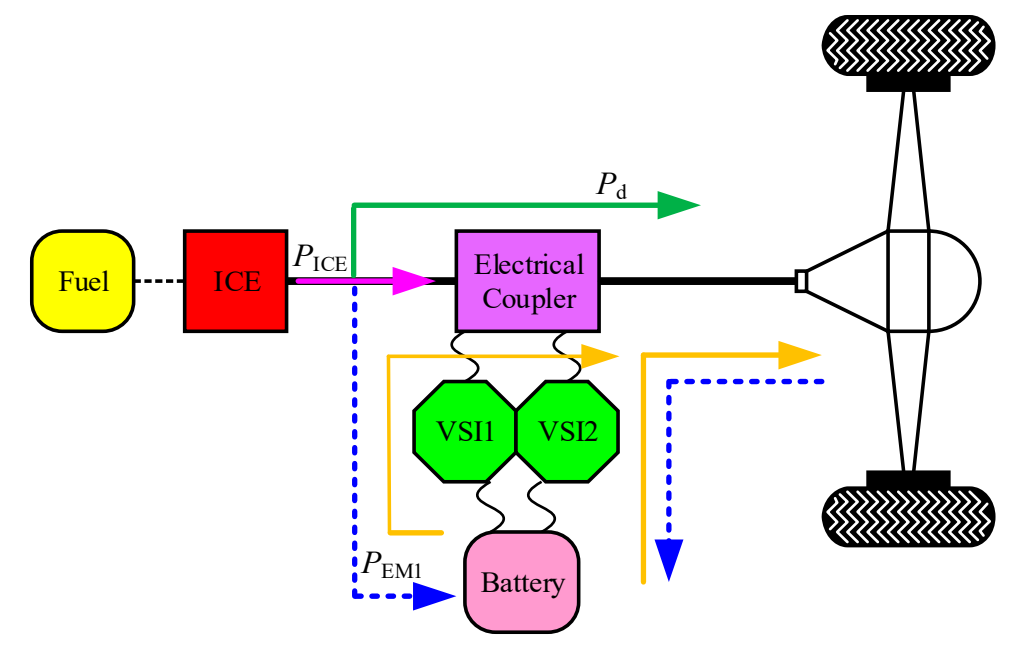

Figure 3. Power split of HEV based on EVT. VSI: voltage source inverter.

Figure 3 shows the operating mechanism of the EVT. The output power of the ICE is divided into two parts by EM1, as shown in Equation (1). In order to describe the process clearly, the $P_{\text {ICE_out, }}$ $P_{\text {EVT_out }}$ are used to express the output power of the ICE and EVT; $T_{\text {ICE }}$ expresses the output torque of the ICE; the $P_{\text {EVT_in }}$ is the input power of the EVT; $P_{\text {Load }}$ is the power required for the road load; $P_{\mathrm{d}}$ represents the power transmitted to the output shaft via the magnetic field. $P_{\mathrm{EM} 1}$ is the output power of EM1.

$$
\begin{aligned}
& P_{\text {ICE_out }}=\omega_{\mathrm{ICE}} \cdot T_{\mathrm{ICE}} \\
& P_{\mathrm{ICE} \_ \text {out }}=P_{\mathrm{EVT} \_ \text {in }}=P_{\mathrm{d}}+P_{\mathrm{EM} 1} \\
& P_{\text {EVT_out }}=P_{\text {Load }}=\omega_{\mathrm{EM} 2}\left(T_{\mathrm{EM} 1}+T_{\mathrm{EM} 2}\right)
\end{aligned}
$$

The $P_{\mathrm{EM} 1}$ is transported to the battery and the stator by using the collector ring, and brush as well as voltage source inverters: voltage source inverter1 (VSI 1) and voltage source inverter2 (VSI 2). The rest of power, i.e., $P_{\mathrm{d}}$, is used to drive the HEV directly.

Due to the fact the inner rotor is mechanically connected to the output shaft of the ICE, the inner rotor has the same speed as the ICE ( $\left.\omega_{\mathrm{ICE}}\right)$ [15]. In addition, the electromagnetic torque difference between the inner and outer rotor is equal to that of ICE in steady case. The output shaft of EVT is connected to final gear of HEVs, i.e., the speed of EM2 $\left(\omega_{\mathrm{EM} 2}\right)$ is determined by the speed of HEV. The output torque of outer rotor combines the torque of EM1 ( $\left.T_{\mathrm{EM} 1}\right)$ and EM2 $\left(T_{\mathrm{EM} 2}\right)$. Table 1 summarizes the operation state of EM1, EM2 and the battery during the power split mode.

Ideally, the battery does not supply the power when $P_{\text {ICE_out }}=P_{\text {Load }}$. However, the battery needs to provide the electrical energy in actual circumstances, because the EVT system has losses under different working conditions. According to the above analysis, the EVT combines the output power of EM1 and EM2 to promote the smooth operation of the HEV under different road conditions. The EVT can improve the system efficiency due to the fact the output power of the ICE is divided into two parts, i.e., $P_{\mathrm{EM} 1}$ and $P_{\mathrm{d}}$. The EM1 works in the generator or the transmission mode to accomplish the power split. The $P_{\mathrm{EM} 1}$ will make the ICE independent of the loads and operate in the optimum efficiency region, which is treated as the series hybrid power system. 
Table 1. Design parameters of EM1 and EM2.

\begin{tabular}{ccccc}
\hline $\begin{array}{c}\text { Power } \\
\text { Relationship }\end{array}$ & $\begin{array}{c}\text { Speed } \\
\text { Relationship }\end{array}$ & EM1 & EM2 & Battery \\
\cline { 1 - 3 }$P_{\text {ICE_out }}=P_{\text {Load }}$ & $\omega_{\text {ICE }}>\omega_{\text {EM2 }}$ & generation operation & electric operation & \\
\cline { 2 - 4 } & $\omega_{\text {ICE }}=\omega_{\mathrm{EM} 2}$ & \multicolumn{2}{c}{ ICE direct-driven mode } & stop operation \\
\cline { 2 - 4 } & $\omega_{\text {ICE }}<\omega_{\mathrm{EM} 2}$ & electric operation & generation operation & \\
\hline$P_{\text {ICE_out }}<P_{\text {Load }}$ & - & generation operation & electric operation & operation \\
\hline$P_{\text {ICE_out }}>P_{\text {Load }}$ & - & generation operation & electric operation & $\begin{array}{c}\text { operation and } \\
\text { charged by EM1 }\end{array}$ \\
\hline
\end{tabular}

The electromagnetic energy $P_{\mathrm{d}}$ has the properties of high efficiency, which resembles the parallel hybrid power system. However, the $P_{\mathrm{d}}$ is transferred to the outer rotor by the electromagnetic coupling effect between electromagnetic fields of EM1 and EM2. The high electromagnetic coupling degree between EM1 and EM2 will weaken the output torque, causing torque ripple, impeding cooling, and decreasing the dynamic performances of the EVT.

\section{Torque Mathematical Model of EVT in ABC Three-Phase Coordinate System}

To analyze the factors that affect the output torque stability during EVT operation, the torque mathematical model of the EVT based on virtual displacement method is proposed. Ignoring the magnetic circuit saturation, iron consumption, windings parameters changing with the temperature, and the self-inductance coefficient of each winding is constant, then the sketch map of the EVT in a three-phase coordinate system is shown in Figure 4.

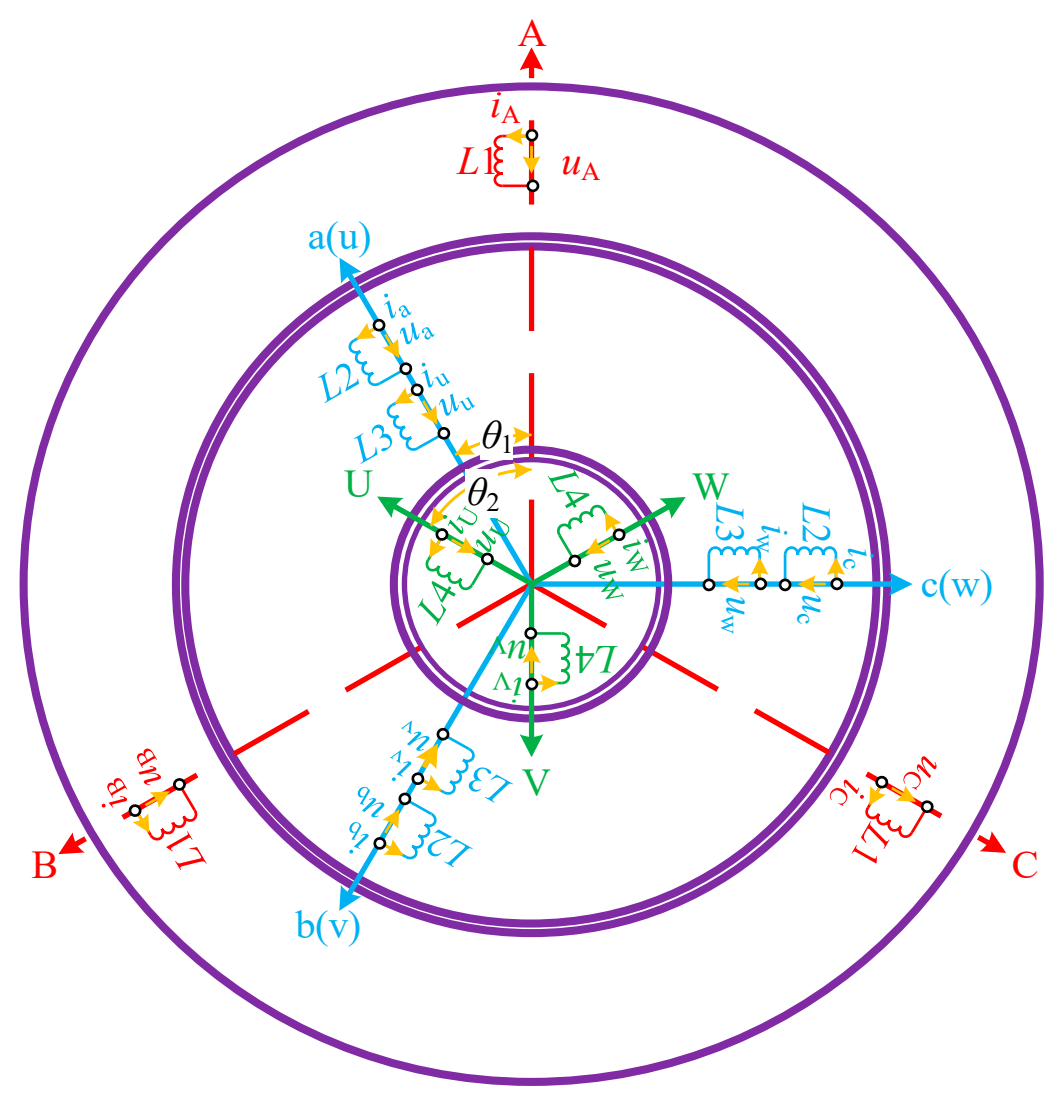

Figure 4. Sketch map of EVT in the ABC three-phase coordinate system. 
In Figure 4, the equivalent windings of stator, outer rotor and inner rotor are converted into the stator side to simplify the analysis, and the electromagnetic relationship in the EVT remains unchanged. The stator windings are $\mathrm{Y}$ connected and represented by A, B, C., and the inner rotor windings are also $\mathrm{Y}$ connected and represented by $\mathrm{U}, \mathrm{V}, \mathrm{W}$. The equivalent windings of outer squirrel-cage are expressed by $a, b$ and $c$. The equivalent windings of the inner squirrel-cage are expressed by $u, v$ and $w$. The relationship of self-inductance and mutual-inductance between stator, outer rotor and inner rotor is shown in Figure 5.

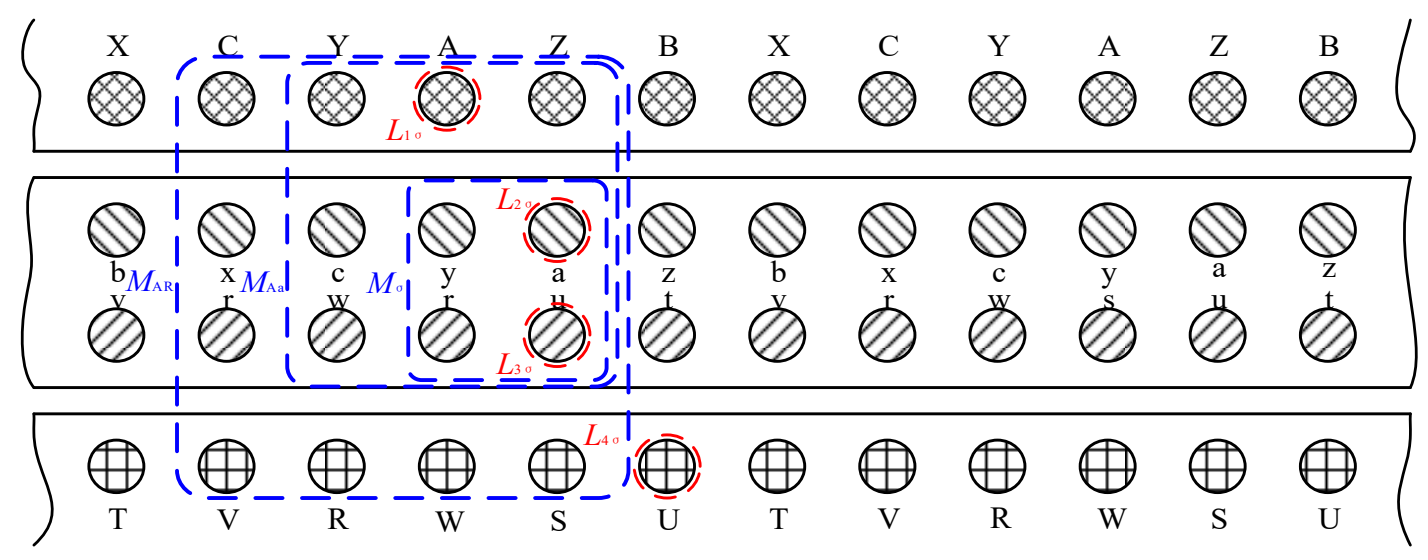

Figure 5. Relationship of inductance and mutual-inductance.

To simplify the equations, we used $L_{1}-L_{4}$ and $M_{1}-M_{6}$ to describe the sum of self-inductance and mutual-inductance, as shown in Equations (2) and (3), where "' " express that the squirrel-cages have the same turns as the stator windings after the winding reduction:

$$
\begin{gathered}
\left\{\begin{array}{l}
L_{1}=L_{1 \sigma}+M_{\mathrm{Aa}}+M_{\mathrm{AR}}=L_{1 \sigma}+L_{\mathrm{ms}} \\
L_{2}=L_{2 \sigma}+M_{\mathrm{Aa}}+M_{\mathrm{AR}}+M_{\sigma}=L_{2 \sigma}+L_{\mathrm{ms}}^{\prime} \\
L_{3}=L_{3 \sigma}+M_{\mathrm{Aa}}+M_{\mathrm{AR}}+M_{\sigma}=L_{3 \sigma}+L_{\mathrm{ms}}^{\prime} \\
L_{4}=L_{4 \sigma}+M_{\mathrm{AR}}
\end{array}\right. \\
\left\{\begin{array}{l}
M_{1}=\frac{1}{2}\left(M_{\mathrm{Aa}}+M_{\mathrm{AR}}\right)=\frac{1}{2} L_{\mathrm{ms}} \\
M_{2}=M_{3}=M_{4}=M_{5}=\frac{1}{2}\left(M_{\mathrm{Aa}}+M_{\mathrm{AR}}+M_{\sigma}\right)=\frac{1}{2} L_{\mathrm{ms}}^{\prime} \\
M_{6}=\frac{1}{2} M_{\mathrm{AR}}
\end{array}\right.
\end{gathered}
$$

Then the voltage equations of stator $\left(u_{\mathrm{A}}, u_{\mathrm{B}}, u_{\mathrm{C}}\right)$, outer squirrel-cage $\left(u_{\mathrm{a}}, u_{\mathrm{b}}, u_{\mathrm{c}}\right)$, inner squirrel-cage $\left(u_{\mathrm{u}}, u_{\mathrm{v}}, u_{\mathrm{W}}\right)$ and inner rotor $\left(u_{\mathrm{U}}, u_{\mathrm{V}}, u_{\mathrm{W}}\right)$ in the ABC three-phase coordinate system are shown in Equations (4)-(7):

$$
\left\{\begin{aligned}
u_{\mathrm{A}}= & R_{1} i_{\mathrm{A}}+L_{1} \frac{d i_{\mathrm{A}}}{d t}-M_{1} \frac{d i_{\mathrm{B}}}{d t}-M_{1} \frac{d i_{\mathrm{C}}}{d t}+\left(M_{\mathrm{Aa}}+M_{\mathrm{AR}}\right) \\
& \cdot \frac{d}{d t}\left[i_{\mathrm{a}} \cos \theta_{1}+i_{\mathrm{b}} \cos \left(\theta_{1}+120^{\circ}\right)+i_{\mathrm{C}} \cos \left(\theta_{1}-120^{\circ}\right)+i_{\mathrm{u}} \cos \theta_{1}\right. \\
& \left.+i_{\mathrm{v}} \cos \left(\theta_{1}+120^{\circ}\right)+i_{\mathrm{W}} \cos \left(\theta_{1}-120^{\circ}\right)\right] \\
& +M_{\mathrm{AR}} \frac{d}{d t}\left[i_{\mathrm{U}} \cos \theta_{2}+i_{\mathrm{V}} \cos \left(\theta_{2}+120^{\circ}\right)+i_{\mathrm{W}} \cos \left(\theta_{2}-120^{\circ}\right)\right] \\
u_{\mathrm{B}}= & R_{1} i_{\mathrm{B}}-M_{1} \frac{d i_{\mathrm{A}}}{d t}+L_{1} \frac{d i_{\mathrm{B}}}{d t}-M_{1} \frac{d i_{\mathrm{C}}}{d t}+\left(M_{\mathrm{Aa}}+M_{\mathrm{AR}}\right) \\
& \cdot \frac{d}{d t}\left[i_{\mathrm{a}} \cos \left(\theta_{1}-120^{\circ}\right)+i_{\mathrm{b}} \cos \theta_{1}+i_{\mathrm{c}} \cos \left(\theta_{1}+120^{\circ}\right)+i_{\mathrm{u}} \cos \left(\theta_{1}-120^{\circ}\right)\right. \\
& \left.+i_{\mathrm{V}} \cos \theta_{1}+i_{\mathrm{W}} \cos \left(\theta_{1}+120^{\circ}\right)\right] \\
& +M_{\mathrm{AR}} \frac{d}{d t}\left[i_{\mathrm{U}} \cos \left(\theta_{2}-120^{\circ}\right)+i_{\mathrm{V}} \cos \theta_{2}+i_{\mathrm{W}} \cos \left(\theta_{2}+120^{\circ}\right)\right] \\
& R_{1} i_{\mathrm{C}}-M_{1} \frac{d i_{\mathrm{A}}}{d t}-M_{1} \frac{d i_{\mathrm{B}}}{d t}+L_{1} \frac{d i_{\mathrm{C}}}{d t}+\left(M_{\mathrm{Aa}}+M_{\mathrm{AR}}\right) \\
& \cdot \frac{d}{d t}\left[i_{\mathrm{a}} \cos \left(\theta_{1}+120^{\circ}\right)+i_{\mathrm{b}} \cos \left(\theta_{1}-120^{\circ}\right)+i_{\mathrm{C}} \cos \theta_{1}+i_{\mathrm{u}} \cos \left(\theta_{1}+120^{\circ}\right)\right. \\
& \left.+i_{\mathrm{v}} \cos \left(\theta_{1}-120^{\circ}\right)+i_{\mathrm{W}} \cos \theta_{1}\right] \\
& +M_{\mathrm{AR}} \frac{d}{d t}\left[i_{\mathrm{U}} \cos \left(\theta_{2}+120^{\circ}\right)+i_{\mathrm{V}} \cos \left(\theta_{2}-120^{\circ}\right)+i_{\mathrm{W}} \cos \theta_{2}\right]
\end{aligned}\right.
$$




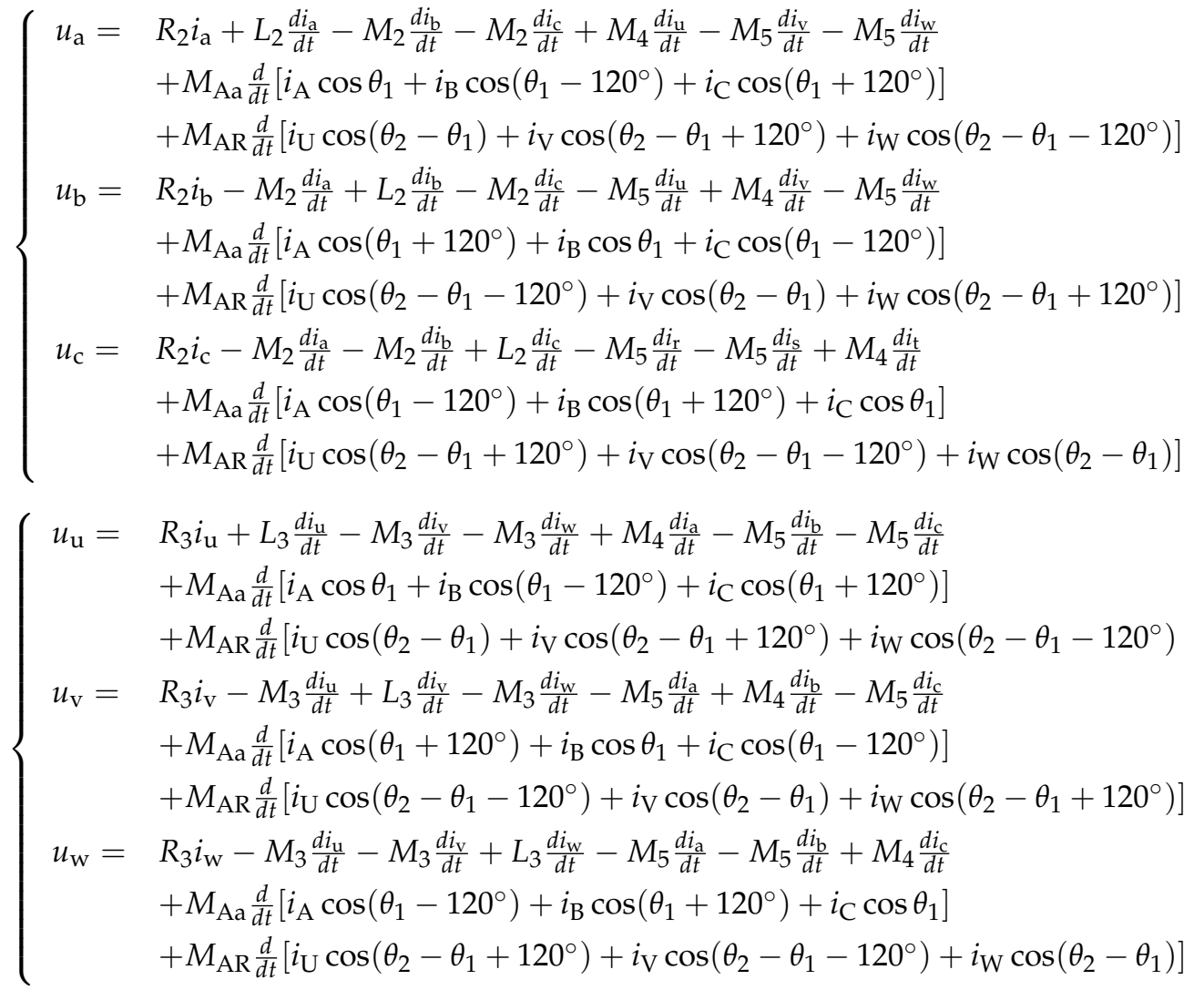

$$
\begin{aligned}
& \left\{\begin{aligned}
u_{\mathrm{U}}= & R_{4} i_{\mathrm{U}}+L_{4} \frac{d i_{\mathrm{U}}}{d t}-M_{6} \frac{d i_{\mathrm{S}}}{d t}-M_{6} \frac{d i_{\mathrm{T}}}{d t} \\
& +M_{\mathrm{AR}} \frac{d}{d t}\left[i_{\mathrm{A}} \cos \theta_{2}+i_{\mathrm{B}} \cos \left(\theta_{2}-120^{\circ}\right)+i_{\mathrm{C}} \cos \left(\theta_{2}+120^{\circ}\right)\right] \\
& +M_{\mathrm{AR}} \frac{d}{d t}\left[\left(i_{\mathrm{a}}+i_{\mathrm{u}}\right) \cos \left(\theta_{2}-\theta_{1}\right)+\left(i_{\mathrm{b}}+i_{\mathrm{V}}\right) \cos \left(\theta_{2}-\theta_{1}-120^{\circ}\right)\right. \\
& \left.+\left(i_{\mathrm{C}}+i_{\mathrm{W}}\right) \cos \left(\theta_{2}-\theta_{1}+120^{\circ}\right)\right] \\
u_{\mathrm{V}}= & R_{4} i_{\mathrm{V}}-M_{6} \frac{d i_{\mathrm{U}}}{d t}+L_{4} \frac{d i_{\mathrm{V}}}{d t}-M_{6} \frac{d i_{\mathrm{W}}}{d t} \\
& +M_{\mathrm{AR}} \frac{d}{d t}\left[i_{\mathrm{A}} \cos \left(\theta_{2}+120^{\circ}\right)+i_{\mathrm{B}} \cos \theta_{2}+i_{\mathrm{C}} \cos \left(\theta_{2}-120^{\circ}\right)\right] \\
& +M_{\mathrm{AR}} \frac{d}{d t}\left[\left(i_{\mathrm{a}}+i_{\mathrm{u}}\right) \cos \left(\theta_{2}-\theta_{1}+120^{\circ}\right)+\left(i_{\mathrm{b}}+i_{\mathrm{V}}\right) \cos \left(\theta_{2}-\theta_{1}\right)\right. \\
& +\left(i_{\mathrm{c}}+i_{\mathrm{W}}\right) \cos \left(\theta_{2}-\theta_{1}-120^{\circ}\right) \\
u_{\mathrm{W}}= & R_{4} i_{\mathrm{W}}-M_{6} \frac{d i_{\mathrm{U}}}{d t}-M_{6} \frac{d i_{\mathrm{V}}}{d t}+L_{4} \frac{d i_{\mathrm{W}}}{d t} \\
& +M_{\mathrm{AR}} \frac{d}{d t}\left[i_{\mathrm{A}} \cos \left(\theta_{2}-120^{\circ}\right)+i_{\mathrm{B}} \cos \left(\theta_{2}+120^{\circ}\right)+i_{\mathrm{C}} \cos \theta_{2}\right] \\
& +M_{\mathrm{AR}} \frac{d}{d t}\left[\left(i_{\mathrm{a}}+i_{\mathrm{u}}\right) \cos \left(\theta_{2}-\theta_{1}-120^{\circ}\right)+\left(i_{\mathrm{b}}+i_{\mathrm{V}}\right) \cos \left(\theta_{2}-\theta_{1}+120^{\circ}\right)\right. \\
& +\left(i_{\mathrm{c}}+i_{\mathrm{W}}\right) \cos \left(\theta_{2}-\theta_{1}\right)
\end{aligned}\right.
\end{aligned}
$$

The matrix expression of flux linkage $(\Psi)$ of the stator, outer rotor and inner rotor is:

$$
\boldsymbol{\Psi}=\left[\begin{array}{llll}
\boldsymbol{L}_{\mathrm{ss}} & \boldsymbol{L}_{\mathrm{sr} 1} & \boldsymbol{L}_{\mathrm{sr} 2} & \boldsymbol{L}_{\mathrm{sr} 3} \\
\boldsymbol{L}_{\mathrm{r} 1 \mathrm{~s}} & \boldsymbol{L}_{\mathrm{r} 1 \mathrm{r} 1} & \boldsymbol{L}_{\mathrm{r} 1 \mathrm{r} 2} & \boldsymbol{L}_{\mathrm{r} 1 \mathrm{r} 3} \\
\boldsymbol{L}_{\mathrm{r} 2 \mathrm{~s}} & \boldsymbol{L}_{\mathrm{r} 2 \mathrm{r} 1} & \boldsymbol{L}_{\mathrm{r} 2 \mathrm{r} 2} & \boldsymbol{L}_{\mathrm{r} 2 \mathrm{r} 3} \\
\boldsymbol{L}_{\mathrm{r} 3 \mathrm{~s}} & \boldsymbol{L}_{\mathrm{r} 3 \mathrm{r} 1} & \boldsymbol{L}_{\mathrm{r} 3 \mathrm{r} 2} & \boldsymbol{L}_{\mathrm{r} 3 \mathrm{r} 3}
\end{array}\right]\left[\begin{array}{l}
i_{\mathrm{s}} \\
i_{\mathrm{r} 1} \\
i_{\mathrm{r} 2} \\
i_{\mathrm{r} 3}
\end{array}\right]
$$


where:

$$
\begin{aligned}
& \boldsymbol{L}_{\mathrm{ss}}=\left[\begin{array}{ccc}
L_{1} & -M_{1} & -M_{1} \\
-M_{1} & L_{1} & -M_{1} \\
-M_{1} & -M_{1} & L_{1}
\end{array}\right] \quad \boldsymbol{L}_{\mathrm{r} 1 \mathrm{r} 1}=\left[\begin{array}{ccc}
L_{2} & -M_{2} & -M_{2} \\
-M_{2} & L_{2} & -M_{2} \\
-M_{2} & -M_{2} & L_{2}
\end{array}\right] \\
& \boldsymbol{L}_{\mathrm{r} 2 \mathrm{r} 2}=\left[\begin{array}{ccc}
L_{3} & -M_{3} & -M_{3} \\
-M_{3} & L_{3} & -M_{3} \\
-M_{3} & -M_{3} & L_{3}
\end{array}\right] \quad \boldsymbol{L}_{\mathrm{r} 3 \mathrm{r} 3}=\left[\begin{array}{ccc}
L_{4} & -M_{6} & -M_{6} \\
-M_{6} & L_{4} & -M_{6} \\
-M_{6} & -M_{6} & L_{4}
\end{array}\right] \\
& \boldsymbol{L}_{\mathrm{sr} 1}=\boldsymbol{L}_{\mathrm{r} 1 \mathrm{~s}}^{\mathrm{T}}=L_{\mathrm{sr} 2}=\boldsymbol{L}_{\mathrm{r} 2 \mathrm{~s}}^{\mathrm{T}}=\boldsymbol{L}_{\mathrm{m}}\left[\begin{array}{ccc}
\cos \theta_{1} & \cos \left(\theta_{1}+120^{\circ}\right) & \cos \left(\theta_{1}-120^{\circ}\right) \\
\cos \left(\theta_{1}-120^{\circ}\right) & \cos \theta_{1} & \cos \left(\theta_{1}+120^{\circ}\right) \\
\cos \left(\theta_{1}+120^{\circ}\right) & \cos \left(\theta_{1}-120^{\circ}\right) & \cos \theta_{1}
\end{array}\right] \\
& \boldsymbol{L}_{\mathrm{sr} 3}=\boldsymbol{L}_{\mathrm{r} 3 \mathrm{~s}}^{\mathrm{T}}=M_{\mathrm{AR}}\left[\begin{array}{ccc}
\cos \theta_{2} & \cos \left(\theta_{2}+120^{\circ}\right) & \cos \left(\theta_{2}-120^{\circ}\right) \\
\cos \left(\theta_{2}-120^{\circ}\right) & \cos \theta_{2} & \cos \left(\theta_{2}+120^{\circ}\right) \\
\cos \left(\theta_{2}+120^{\circ}\right) & \cos \left(\theta_{2}-120^{\circ}\right) & \cos \theta_{2}
\end{array}\right] \\
& \boldsymbol{L}_{\mathrm{r} 1 \mathrm{r} 2}=\boldsymbol{L}_{\mathrm{r} 2 \mathrm{r} 1}^{\mathrm{T}}=M_{4}\left[\begin{array}{ccc}
1 & -\frac{1}{2} & -\frac{1}{2} \\
-\frac{1}{2} & 1 & -\frac{1}{2} \\
-\frac{1}{2} & -\frac{1}{2} & 1
\end{array}\right]=\left[\begin{array}{ccc}
M_{4} & -M_{5} & -M_{5} \\
-M_{5} & M_{4} & -M_{5} \\
-M_{5} & -M_{5} & M_{4}
\end{array}\right] \\
& L_{\mathrm{r} 1 \mathrm{r} 3}=\boldsymbol{L}_{\mathrm{r} 3 \mathrm{r} 1}^{\mathrm{T}}=\boldsymbol{L}_{\mathrm{r} 2 \mathrm{r} 3}=\boldsymbol{L}_{\mathrm{r} 3 \mathrm{r} 2}^{\mathrm{T}} \\
& =\boldsymbol{M}_{\mathrm{AR}}\left[\begin{array}{ccc}
\cos \left(\theta_{2}-\theta_{1}\right) & \cos \left(\theta_{2}-\theta_{1}+120^{\circ}\right) & \cos \left(\theta_{2}-\theta_{1}-120^{\circ}\right) \\
\cos \left(\theta_{2}-\theta_{1}-120^{\circ}\right) & \cos \left(\theta_{2}-\theta_{1}\right) & \cos \left(\theta_{2}-\theta_{1}+120^{\circ}\right) \\
\cos \left(\theta_{2}-\theta_{1}+120^{\circ}\right) & \cos \left(\theta_{2}-\theta_{1}-120^{\circ}\right) & \cos \left(\theta_{2}-\theta_{1}\right)
\end{array}\right]
\end{aligned}
$$

According to the virtual displacement method, the electromagnetic torque is equal to the partial derivative of magnetic co-energy to the rotor mechanical angle. The magnetic co-energy in the system under linear condition is:

$$
W_{\mathrm{m}}^{\prime}=\frac{1}{2} i^{T} \boldsymbol{\Psi}=\frac{1}{2} i^{T} \boldsymbol{N} \phi=\frac{1}{2} i^{T} \boldsymbol{L} \boldsymbol{i}
$$

The relationship of electromagnetic torque between stator, outer rotor and inner rotor is given in Figure 6. The electromagnetic torques generated by the inner rotor on the outer rotor $\left(T_{12}\right)$ and generated by the outer rotor on the inner rotor $\left(T_{21}\right)$ are a pair of acting forces and reaction forces, so the $T_{12}$ is equal to $T_{21}$. Then the electromagnetic torque of outer rotor $\left(T_{\mathrm{r} 1 \mathrm{r} 2 \mathrm{sr} 3}\right)$ and inner rotor $\left(T_{\mathrm{r} 3 \mathrm{sr} 1 \mathrm{r} 2}\right)$ can be obtained as follows:

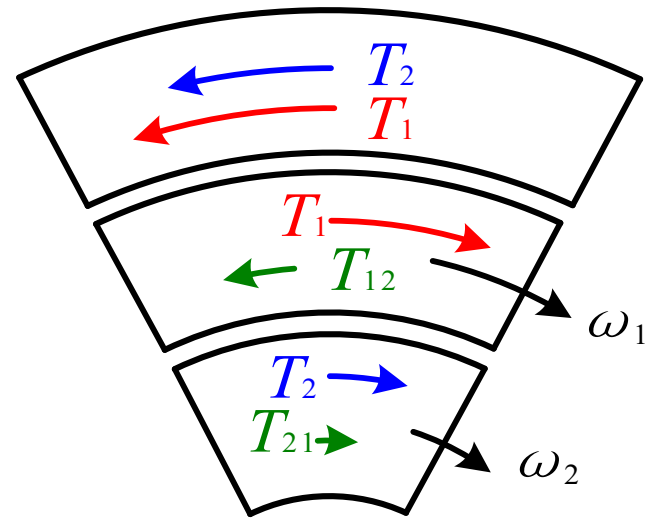

Figure 6. Torque relationship in EVT. 


$$
\begin{aligned}
& T_{\mathrm{r} 1 \mathrm{r} 2 \mathrm{sr} 3}=T_{1}-T_{12}=p_{\mathrm{n}}\left[\frac{\partial W_{\mathrm{m}}^{\prime}}{\partial \theta_{1}}-\frac{\partial W_{\mathrm{m}}^{\prime}}{\partial\left(\theta_{2}-\theta_{1}\right)}\right] \\
& =p_{\mathrm{n}}\left(M_{\mathrm{Aa}}+M_{\mathrm{AR}}\right)\left\{i _ { \mathrm { A } } \left[\left(i_{\mathrm{a}}+i_{\mathrm{u}}\right) \sin \theta_{1}+\left(i_{\mathrm{b}}+i_{\mathrm{V}}\right) \sin \left(\theta_{1}+120^{\circ}\right)\right.\right. \\
& \left.+\left(i_{\mathrm{c}}+i_{\mathrm{w}}\right) \sin \left(\theta_{1}-120^{\circ}\right)\right]+i_{\mathrm{B}}\left[\left(i_{\mathrm{a}}+i_{\mathrm{u}}\right) \sin \left(\theta_{1}-120^{\circ}\right)+\left(i_{\mathrm{b}}+i_{\mathrm{v}}\right) \sin \theta_{1}\right. \\
& \left.+\left(i_{\mathrm{c}}+i_{\mathrm{w}}\right) \sin \left(\theta_{1}+120^{\circ}\right)\right]+i_{\mathrm{C}}\left[\left(i_{\mathrm{a}}+i_{\mathrm{u}}\right) \sin \left(\theta_{1}+120^{\circ}\right)\right. \\
& \left.\left.+\left(i_{\mathrm{b}}+i_{\mathrm{v}}\right) \sin \left(\theta_{1}-120^{\circ}\right)+\left(i_{\mathrm{c}}+i_{\mathrm{w}}\right) \sin \theta_{1}\right]\right\} \\
& -p_{\mathrm{n}} M_{\mathrm{AR}}\left\{i _ { \mathrm { U } } \left[\left(i_{\mathrm{a}}+i_{\mathrm{u}}\right) \sin \left(\theta_{2}-\theta_{1}\right)+\left(i_{\mathrm{b}}+i_{\mathrm{V}}\right) \sin \left(\theta_{2}-\theta_{1}+120^{\circ}\right)\right.\right. \\
& \left.+\left(i_{\mathrm{c}}+i_{\mathrm{w}}\right) \sin \left(\theta_{2}-\theta_{1}-120^{\circ}\right)\right]+i_{\mathrm{V}}\left[\left(i_{\mathrm{a}}+i_{\mathrm{u}}\right) \sin \left(\theta_{2}-\theta_{1}-120^{\circ}\right)\right. \\
& \left.+\left(i_{\mathrm{b}}+i_{\mathrm{v}}\right) \sin \left(\theta_{2}-\theta_{1}\right)+\left(i_{\mathrm{c}}+i_{\mathrm{w}}\right) \sin \left(\theta_{2}-\theta_{1}+120^{\circ}\right)\right] \\
& +i_{\mathrm{W}}\left[\left(i_{\mathrm{a}}+i_{\mathrm{u}}\right) \sin \left(\theta_{2}-\theta_{1}+120^{\circ}\right)+\left(i_{\mathrm{b}}+i_{\mathrm{v}}\right) \sin \left(\theta_{2}-\theta_{1}-120^{\circ}\right)\right. \\
& \left.\left.+\left(i_{\mathrm{c}}+i_{\mathrm{w}}\right) \sin \left(\theta_{2}-\theta_{1}\right)\right]\right\} \\
& T_{\mathrm{r} 3 \mathrm{sr} 1 \mathrm{r} 2}=T_{2}+T_{21}=p_{\mathrm{n}}\left[\frac{\partial W_{\mathrm{m}}^{\prime}}{\partial \theta_{2}}+\frac{\partial W_{\mathrm{m}}^{\prime}}{\partial\left(\theta_{2}-\theta_{1}\right)}\right] \\
& =p_{\mathrm{n}} M_{\mathrm{AR}}\left\{\left(i_{\mathrm{A}} i_{\mathrm{U}}+i_{\mathrm{B}} i_{\mathrm{V}}+i_{\mathrm{C}} i_{\mathrm{W}}\right) \sin \theta_{2}+\left(i_{\mathrm{A}} i_{\mathrm{U}}+i_{\mathrm{B}} i_{\mathrm{V}}+i_{\mathrm{C}} i_{\mathrm{W}}\right)\right. \\
& \left.\cdot \sin \left(\theta_{2}+120^{\circ}\right)+\left(i_{\mathrm{A}} i_{\mathrm{W}}+i_{\mathrm{B}} i_{\mathrm{U}}+i_{\mathrm{C}} i_{\mathrm{V}}\right) \sin \left(\theta_{2}-120^{\circ}\right)\right\}+p_{\mathrm{n}} M_{\mathrm{AR}} \\
& \cdot\left\{i _ { \mathrm { U } } \left[\left(i_{\mathrm{a}}+i_{\mathrm{u}}\right) \sin \left(\theta_{2}-\theta_{1}\right)+\left(i_{\mathrm{b}}+i_{\mathrm{V}}\right) \sin \left(\theta_{2}-\theta_{1}+120^{\circ}\right)+\left(i_{\mathrm{c}}+i_{\mathrm{W}}\right)\right.\right. \\
& \left.\sin \left(\theta_{2}-\theta_{1}-120^{\circ}\right)\right]+i_{\mathrm{V}}\left[\left(i_{\mathrm{a}}+i_{\mathrm{u}}\right) \sin \left(\theta_{2}-\theta_{1}-120^{\circ}\right)+\left(i_{\mathrm{b}}+i_{\mathrm{V}}\right) \sin \left(\theta_{2}-\theta_{1}\right)\right. \\
& \left.+\left(i_{\mathrm{c}}+i_{\mathrm{w}}\right) \sin \left(\theta_{2}-\theta_{1}+120^{\circ}\right)\right]+i_{\mathrm{W}}\left[\left(i_{\mathrm{a}}+i_{\mathrm{u}}\right) \sin \left(\theta_{2}-\theta_{1}+120^{\circ}\right)\right. \\
& \left.\left.+\left(i_{\mathrm{b}}+i_{\mathrm{v}}\right) \sin \left(\theta_{2}-\theta_{1}-120^{\circ}\right)+\left(i_{\mathrm{c}}+i_{\mathrm{w}}\right) \sin \left(\theta_{2}-\theta_{1}\right)\right]\right\}
\end{aligned}
$$

where $P_{\mathrm{n}}$ 一the number of pole-pairs of EVT; $\theta_{1}$-the angle between the axis of winding A and winding a; $\theta_{2}$-the angle between the axis of winding $A$ and winding $R ; i_{A}, i_{\mathrm{B}}, i_{C}$ - the current instantaneous value of stator windings; $i_{\mathrm{a}}, i_{\mathrm{b}}, i_{\mathrm{c}}, i_{\mathrm{u}}, i_{\mathrm{v}}, i_{\mathrm{w}}$-the current instantaneous value of outer and inner squirrel-cage; $i_{\mathrm{U}}, i_{\mathrm{V}}, i_{\mathrm{W}}$-the current instantaneous value of inner rotor windings; $R_{1}, R_{2}, R_{3}, R_{4}$ - the total equivalent resistance of stator windings, outer and inner squirrel-cage, inner rotor windings; $L_{1 \sigma}$, $L_{2 \sigma}, L_{3 \sigma}, L_{4 \sigma}$ - the leakage inductance of stator, outer and inner squirrel-cage, inner rotor generated by the leakage flux; $s$ stands for stator parameters, $r_{1}$ and $r_{2}$ express the parameters of inner and outer squirrel cage, respectively, $r_{3}$ is the parameters for inner rotor; $L_{\mathrm{ss}}, L_{\mathrm{r} 1 \mathrm{r} 1}, L_{\mathrm{r} 1 \mathrm{r} 1}, L_{\mathrm{r} 1 \mathrm{r} 1}$ - the phase winding self-inductance of stator windings, outer and inner squirrel-cage, inner rotor windings; $L_{\mathrm{sr} 1}, L_{\mathrm{r} 1 \mathrm{~s}}^{\mathrm{T}}$, $L_{\mathrm{sr} 2}, L_{\mathrm{r} 2 \mathrm{~s}}{ }^{\mathrm{T}}, L_{\mathrm{sr} 3}, L_{\mathrm{r} 3 \mathrm{~s}}{ }^{\mathrm{T}}$ - the mutual-inductance between stator and outer squirrel-cage, inner squirrel cage, inner rotor, respectively; $L_{\mathrm{r} 1 \mathrm{r} 2}, L_{\mathrm{r} 2 \mathrm{r} 1} \mathrm{~T}$ - the mutual-inductance between outer and inner-squirrel cage; $L_{\mathrm{r} 1 \mathrm{r} 3}, L_{\mathrm{r} 3 \mathrm{r} 1}{ }^{\mathrm{T}}$ - the mutual-inductance between outer-squirrel and inner rotor; $L_{\mathrm{r} 2 \mathrm{r} 3}, L_{\mathrm{r} 3 \mathrm{r} 2}{ }^{\mathrm{T}}$ 一the mutual-inductance between inner squirrel and inner rotor; $L_{\mathrm{m}}$-the mutual-inductance between stator, outer rotor when the axis of them coincidence; $M_{\mathrm{Aa}}, M_{\mathrm{AR}}$-the mutual-inductance between stator and outer rotor, inner rotor, $M_{\sigma}$-the leakage mutual-inductance between outer and inner squirrel-cage.

The torque ripple is an important index to evaluate the operation stability of the EVT, which has a great influence on the comfort of HEVs. When the torque ripple frequency coincides with the working frequency, it will cause a resonance phenomenon and affect the output efficiency of the EVT. It can be seen from Equations (11) and (12) that $P_{\mathrm{n}}$ is a constant, the torque of outer rotor is related to the $M_{\mathrm{Aa}}$, $M_{\mathrm{AR}}$, and the torque of inner rotor is associated with the $M_{\mathrm{AR}}$, so the torque ripple is mainly produced in two aspects. Firstly, both of the EM1 and EM2 are composed by the outer rotor. Their magnetic circuits are closed through the outer rotor, which makes the outer rotor easily saturated. When the magnetic field is saturated, the mutual-inductance will be in a nonlinear state. The derivative of the mutual-inductance to the position is not 0 , which does not change linearly and makes the torque of outer rotor ripple serious. Secondly, the output torque cannot change with the symmetric current excitation when the magnetic field is seriously saturated. Both of the torques of outer rotor and stator are related to the instantaneous current of stator, outer rotor and inner rotor, so it is necessary to investigate the influence of different currents on the torque ripple. 
The harms of torque ripple are big electromagnetic noise, poor output torque and control accuracy. The interference between inner and outer magnetic fields can be eliminated from the proper structure parameters, which greatly improves the torque property and reduces the size of the EVT. The next part of the article studies the optimal design of the structure parameters to weaken the coupling degree and enhance the torque stability.

\section{Optimal Design}

There are two magnetic potential sources in the EVT, which complicate the structure and lead to serious coupling. Therefore, the structural parameters of EVT have a great influence on the electromagnetic performance. The mutual-inductance linearity degree changes with the magnetic conductance, which is related to the magnetic circuit length, area and saturation degree. The magnetic circuit length and area are influenced by the slot width, yoke thickness of stator, outer rotor and inner rotor etc. The winding turns and excitation current impact the magnetic co-energy, and have a certain effect on the magnetic field saturation degree. We can optimal design these factors to improve the torque stability. The outer rotor's output torque is composed by that of EM1 and EM2, so we separately analyze their torque ripple to reduce the total ripple.

\subsection{Slot Width}

In order to study the effect of outer rotor yoke thickness, stator yoke height on the torque ripple, the inner and outer diameters of the inner rotor are limited to $61 \mathrm{~mm}$ and $113 \mathrm{~mm}$, respectively. The inner diameter of the outer rotor is chosen as $114 \mathrm{~mm}$. For different slot widths of the inner rotor and stator, the torque ripple percentage curve is calculated. The analysis result is given in Figure 7. As can be seen, an increase in the slot width will add the torque ripple for EM1 and EM2, and due to the fact the distance of the air-gap in the tangential is raised, the magnetic flux leakage and harmonic content in the air-gap are also raised. However, the slot widths of the two machines have no appreciable influence on the torque ripple within the analysis range. The torque ripple percentage only increased by $1.5 \%$ when the slot width changed from $1.6 \mathrm{~mm}$ to $3 \mathrm{~mm}$ of EM1. In EM2, the torque ripple only changes about $3.5 \%$ under slot width is $3.4 \mathrm{~mm}-4.8 \mathrm{~mm}$. Therefore, the torque ripple improvement for EM1 and EM2 is not obvious by optimizing the slot width. Then the effect of other structural parameters on torque ripple are researched.

\subsection{Outer Rotor Outer Diameter}

The design of the yoke thickness of stator and rotor should be able to avoid the phenomenon of electromagnetic super-saturation in them. The outer diameter of the outer rotor influences on the torque ripple of EM1 and EM2 are shown in Figure 8, where it can be seen that the change of the outer rotor size has significant influence on the torque ripple of EM1 and EM2. The rise of the outer rotor size reduces the torque ripple of the two machines until the outer diameter reaches $166 \mathrm{~mm}$. This is because when the outer diameter of outer rotor is increasing, the magnetic fields coupling degree of EM1 and EM2 in the outer rotor will be reduced. The increase of the magnetic circuit linearity results in the decrease of harmonic content in the air-gap, so the torque ripple is weaken. However, the change range of torque ripple is small when the outer diameter of outer rotor increases to a certain degree, namely, more than $166 \mathrm{~mm}$. It shows that the magnetic fields of two machines is in a state of non-interference.

\subsection{Stator Outer Diameter}

The trend of the change of the torque ripple under different stator outer diameters is very similar to that in different outer rotor sizes, as given in Figure 9. The torque ripples of EM1 and EM2 decrease with the increase of stator outer diameter until the diameter reaches $228 \mathrm{~mm}$. Nevertheless, when the diameter exceeds $228 \mathrm{~mm}$, the saturation issue becomes non-dominant and the torque ripple percentage is basically stable. 

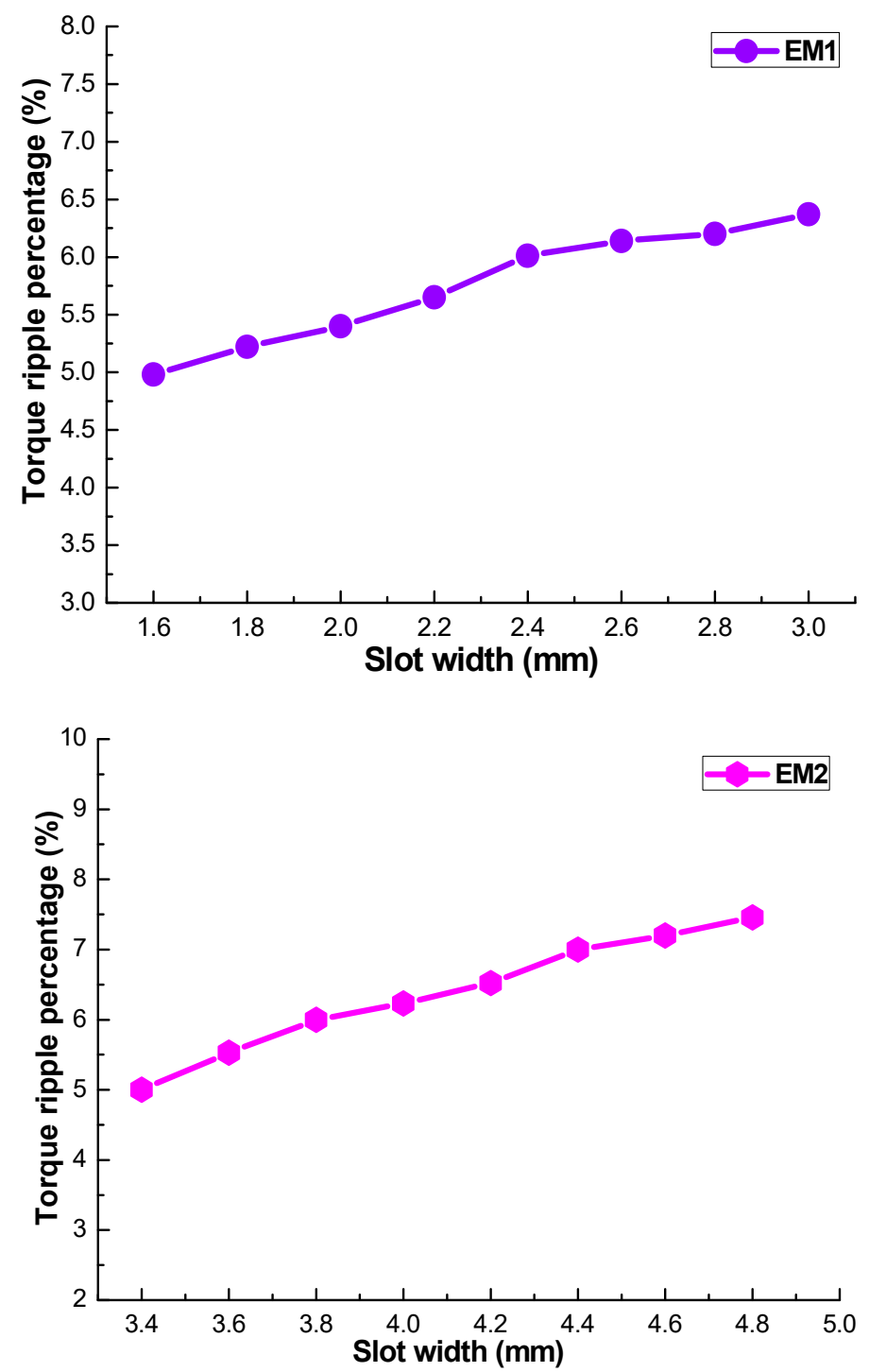

Figure 7. Effect of slot width on the torque ripple for EM1 and EM2.

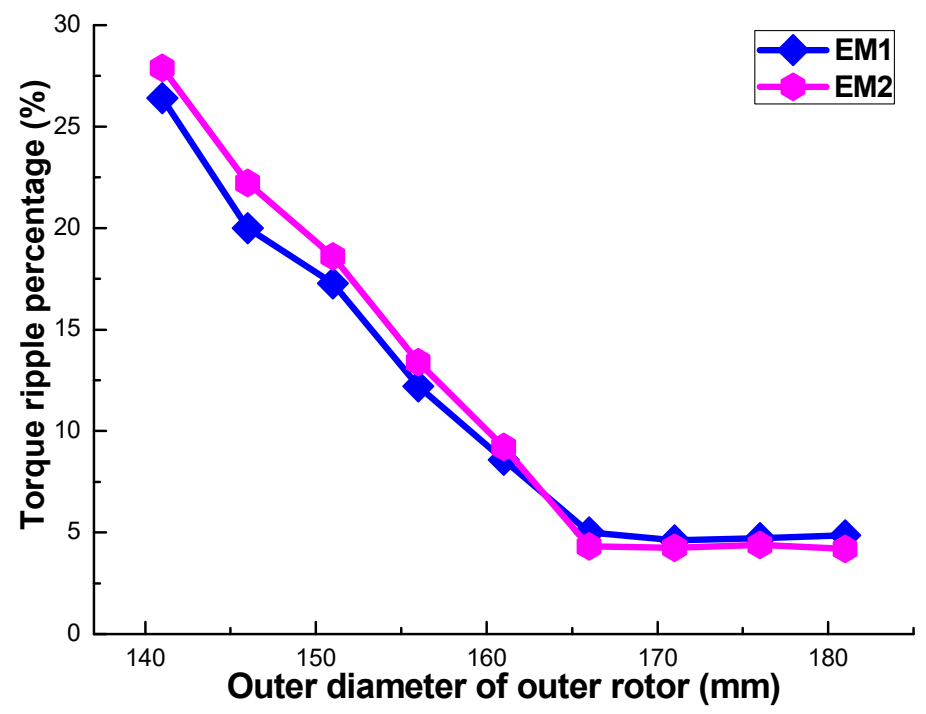

Figure 8. Effect of outer rotor outer diameter on the torque ripple for EM1 and EM2. 


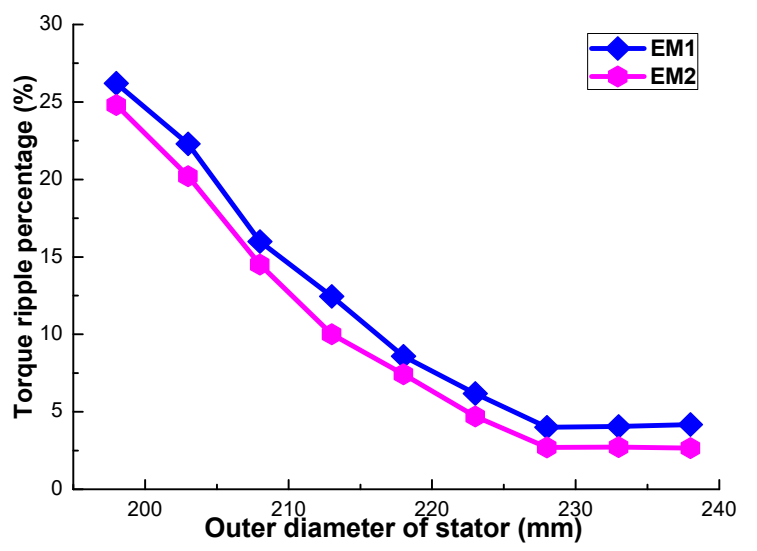

Figure 9. Effect of stator outer diameter on the torque ripple for EM1 and EM2.

\subsection{Winding Turns per Slot}

In order to make the output torque change with the symmetric excitation phase, the winding turns of inner rotor and stator are researched to increase the magnetic co-energy properly, which will regulate the instantaneous torque waveform. The number of winding turns per slot of inner rotor are changing from 12 to 36, and that of stator are changing from 8 to 32. The analysis results are given in Figure 10.

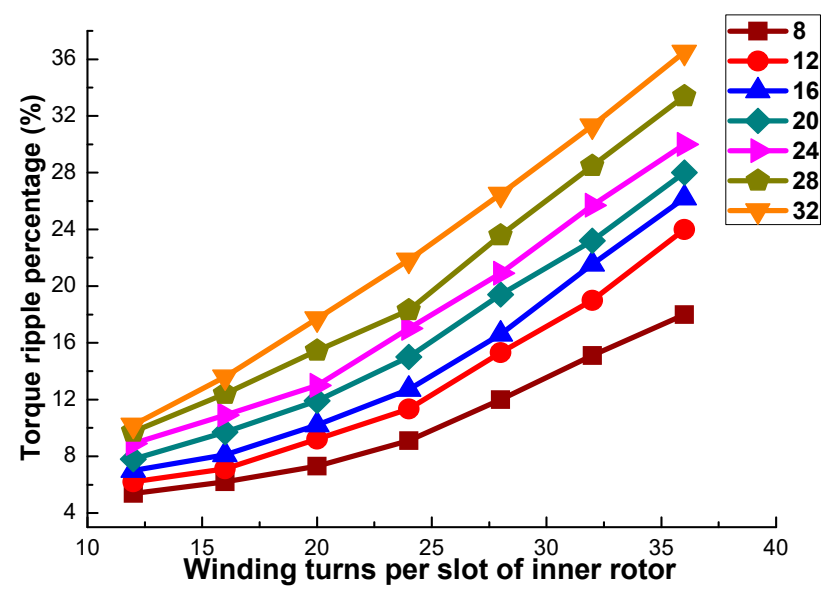

(a)

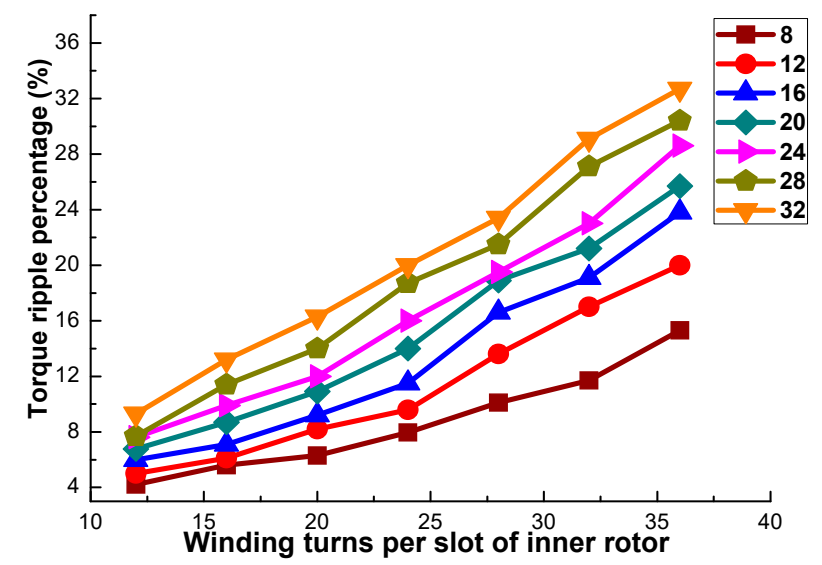

(b)

Figure 10. Effect of winding turns on the torque ripple for (a) EM1; (b) EM2. 
In Figure 10a, the torque ripple of EM1 increases with the rise of winding turns slowly and then rapidly. The turning point appears when the number of winding turns per slot in the inner rotor is 24 . When the number of winding turns per slot in stator is 32, the torque ripple increases linearly with different winding turns per slot in the inner rotor. The slowest increase in torque ripple occurs when the winding turns per slot in the stator is 8 . This because when winding turns are added, magnetic co-energy is also added, which increases the magnetic coupling degree. At this time the output torque cannot be followed by the current change in real time, which makes the torque ripple more and more serious. In Figure 10b, the influence of winding turns on the torque ripple of EM2 is slightly similar to that of EM1. The difference is with the increase of magnetic field saturation, the growth rate of torque ripple for EM2 is less than that of EM1.

\subsection{Excitation Current with Different Amplitude and Initial Phase Angle}

The other method to increase the magnetic co-energy is to analyze the excitation current with different amplitude and initial phase angle. The magneto-motive force of the EVT is provided by the windings of EM1 and EM2, and the magnetic field coupling state in EVT is different under different excitation currents. The effects of excitation current on the torque ripple of EM1 and EM2 are shown in Figure 11.

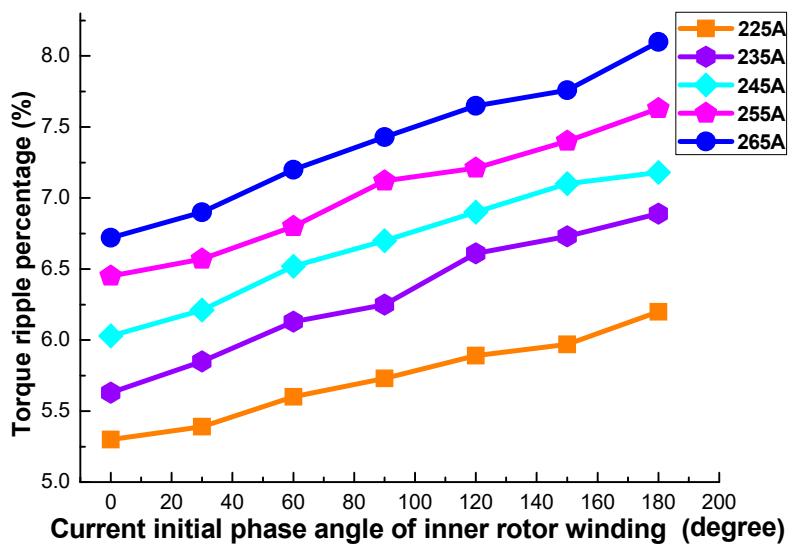

(a)

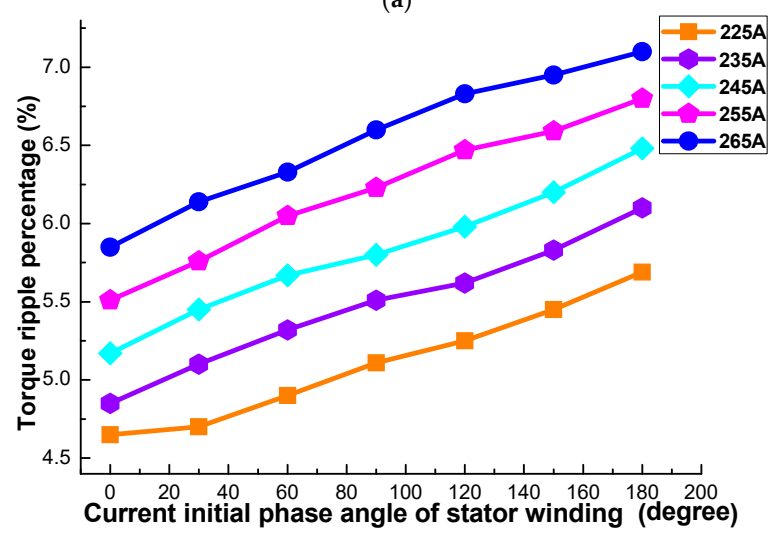

(b)

Figure 11. Effect of excitation current on the torque ripple for (a) EM1; (b) EM2.

The variation range of excitation current initial phase angle is 0 to 180 degree in EM1 and EM2. As can be seen in Figure 11a, the torque ripple of EM1 increases as the current amplitude increases from $225 \mathrm{~A}$ to $265 \mathrm{~A}$. At the same time, if the excitation current amplitude is identical, the magnetic density and coupling degree of EM1 and EM2 will change with the initial phase angle. The torque ripple also adds with the rise of under the same current amplitude. In Figure 11b, the effect of excitation current on the torque properties of EM2 is quite similar to that of EM1. The torque ripple of EM2 increases 
with excitation current between $225 \mathrm{~A}$ to $265 \mathrm{~A}$. This is because the magnetic field coupling state and saturation degree are different when the windings given different excitation currents. The inductance and mutual-inductance of machine are also different. The coupling degree of magnetic circuit has increased, which makes the torque ripple serious and the precise control of EVT becomes more difficult.

In summary, based on the above analysis, it can be seen that to reduce the magnetic field coupling degree, decrease the torque ripple to improve the output torque performance of EVT the following actions are required:

(1) A smaller slot width of stator and inner rotor is expected. However, the slot width of the machine should be a little larger to facilitate coil processing. Generally speaking, the slot width is at least three multiples of the wire diameter. As we choose the wire diameter of inner rotor and stator is $0.65 \mathrm{~mm}$ and $1.3 \mathrm{~mm}$, the slot width of EM1 and EM1 is identified as $2 \mathrm{~mm}$ and the $4 \mathrm{~mm}$, separately;

(2) A larger outer diameter of outer rotor and stator is desirable considering torque ripple, but the torque ripple gradually becomes steady when the size increases to a certain degree. For reducing the size of EVT and convenience for installing in HEVs, the outer diameter of the outer rotor is selected as $166 \mathrm{~mm}$, and the yoke thickness of the outer rotor is $14 \mathrm{~mm}$. The outer diameter of the stator is determined as $223 \mathrm{~mm}$, and its yoke thickness is $14.5 \mathrm{~mm}$;

(3) Fewer winding turns are required to reduce the torque ripple. However, with fewer winding turns, the excitation reactance is smaller, which will increase the excitation current. In order to make the machine need a smaller excitation current, the winding turns per slot in the inner rotor and stator are set at 16 and 8, respectively;

(4) A smaller excitation current amplitude and initial phase angle can reduce the torque ripple. In this optimal design, the excitation current amplitude of EM1 is chosen as $30 \mathrm{~A}$, and initial phase angle is 0 degrees. For EM2, the excitation current amplitude is $90 \mathrm{~A}$, and the initial phase angle also chosen as 0 degrees.

\section{Performance Validation}

In this part, an EVT where EM1 is $15 \mathrm{~kW}$ and EM2 is $30 \mathrm{~kW}$ is designed. According to the above optimization analysis, a FEM model is constructed for the electromagnetic design of the EVT, as shown in Figure 1a. The detailed parameters are given in Table 2.

Table 2. Design parameters of EM1 and EM2.

\begin{tabular}{ccc}
\hline Design Parameters & EM1 & EM2 \\
\hline Rated power $(\mathrm{kW})$ & 15 & 30 \\
Rated current $(\mathrm{A})$ & 225 & 225 \\
Rated speed $(\mathrm{rpm})$ & 5000 & 2400 \\
Number of phase & 3 & 3 \\
Iron core material & DW310-35 & DW310-35 \\
Squirrel-cage material & red copper & red copper \\
Number of slot & 24 & 36 \\
Number of squirrel-cage & 30 & 33 \\
Iron core length (mm) & 90 & 90 \\
Air-gap length (mm) & 0.5 & 0.5 \\
Slot width (mm) & 2 & 4 \\
Squirrel-cage width (mm) & 8 & 8 \\
Squirrel-cage thickness (mm) & 6 & 6 \\
\hline Inner diameter of inner rotor $(\mathrm{mm})$ & 61 \\
Outer diameter of inner rotor $(\mathrm{mm})$ & 113 \\
Inner diameter of outer rotor $(\mathrm{mm})$ & 114 \\
Outer diameter of outer rotor $(\mathrm{mm})$ & 166 \\
Inner diameter of stator $(\mathrm{mm})$ & 167 \\
Outer diameter of stator $(\mathrm{mm})$ & 223 \\
\hline
\end{tabular}


The performance of the EVT after the optimization design is analyzed based on the FEM model and the simulation results are given as follows.

\subsection{Field Distribution}

The magnetic field of the EVT is computed using FEM. The magnetic field density and magnetic flux direction under rated load are plotted in Figure 12. In Figure 12a, most parts of the EVT are in the low magnetic field density state, such as the outer rotor, slot of stator and inner rotor. This indicates that EVT is in a state of unsaturation. The low magnetic circuit saturation degree can not only increase the power density of the EVT, but also effectively restrain the iron loss when driving at high speed. The larger magnetic flux density appears in the stator yoke, inner rotor yoke and tooth. The maximum flux density is no more than $2 \mathrm{~T}$, which is allowable for the iron core material.

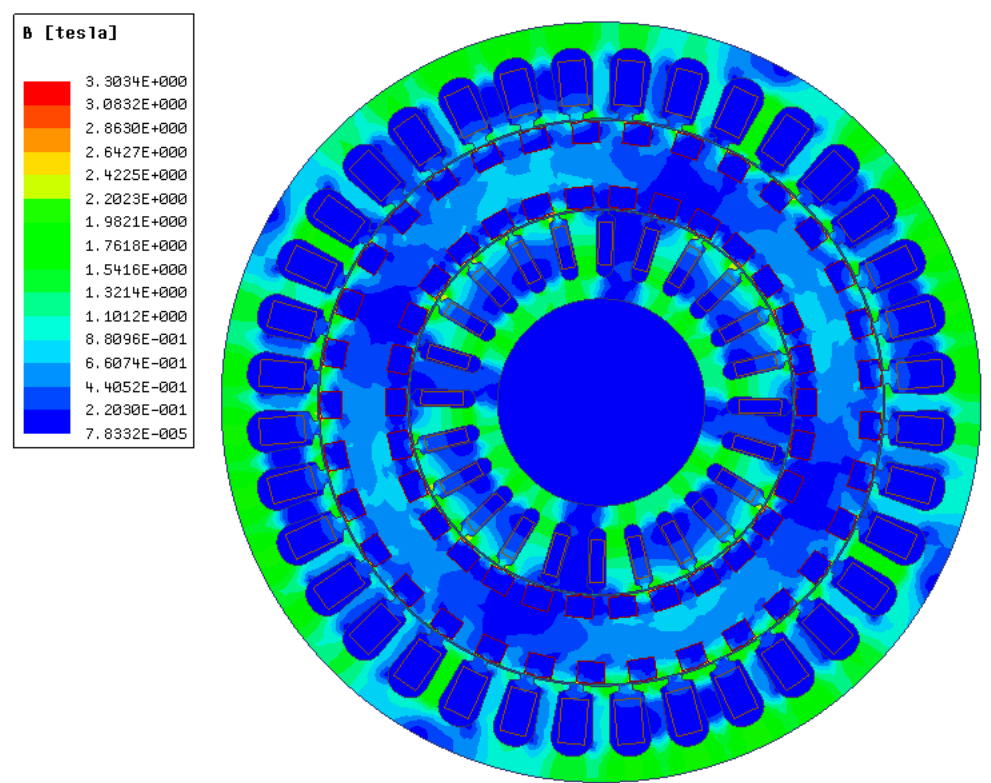

(a)

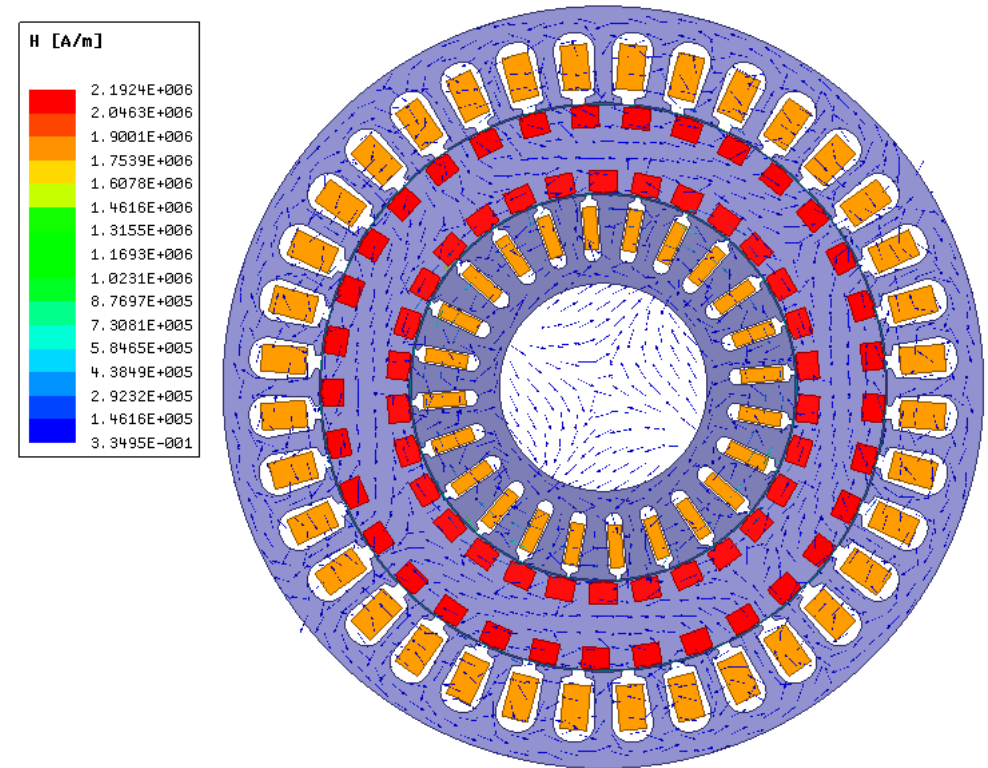

(b)

Figure 12. Magnetic field of EVT: (a) magnetic flux density; (b) magnetic flux direction. 
From Figure $12 \mathrm{~b}$ can be seen the flow path of magnetic flux lines of EM1 and EM2. In EM2, the magnetic flux lines flow in the stator yoke, stator tooth, air-gap, outer rotor yoke, then return to the stator yoke to form a closed magnetic circuit, while in EM1, the closed magnetic circuit is composed by inner rotor yoke and its teeth, air-gap, outer rotor yoke, then return. The magnetic flux lines of the two machines have little interference with each other. Through the above structural optimization design, the distribution of the whole magnetic field in EVT is more reasonable. The magnetic circuit saturation degree and the coupling degree between EM1 and EM2 are reduced, so the working losses of the EVT are reduced under complicated conditions. However, there are some leakage fluxes in the stator and inner rotor slot, which do not participate in the electromagnetic energy transmission and should be further investigated to weaken them.

\subsection{Induced Voltage at Full-Load Operation}

The induced voltage is one of the key factors that decides the operating voltage. The sinusoidal degree of the induced voltage waveform also influences the efficiency, vibration, and noise of the EVT. When the EM1 speed is $5000 \mathrm{rpm}$ and the EM2 is $2400 \mathrm{rpm}$, the induced voltages in the stator windings and inner rotor windings are calculated by FEM, as shown in Figure 13.

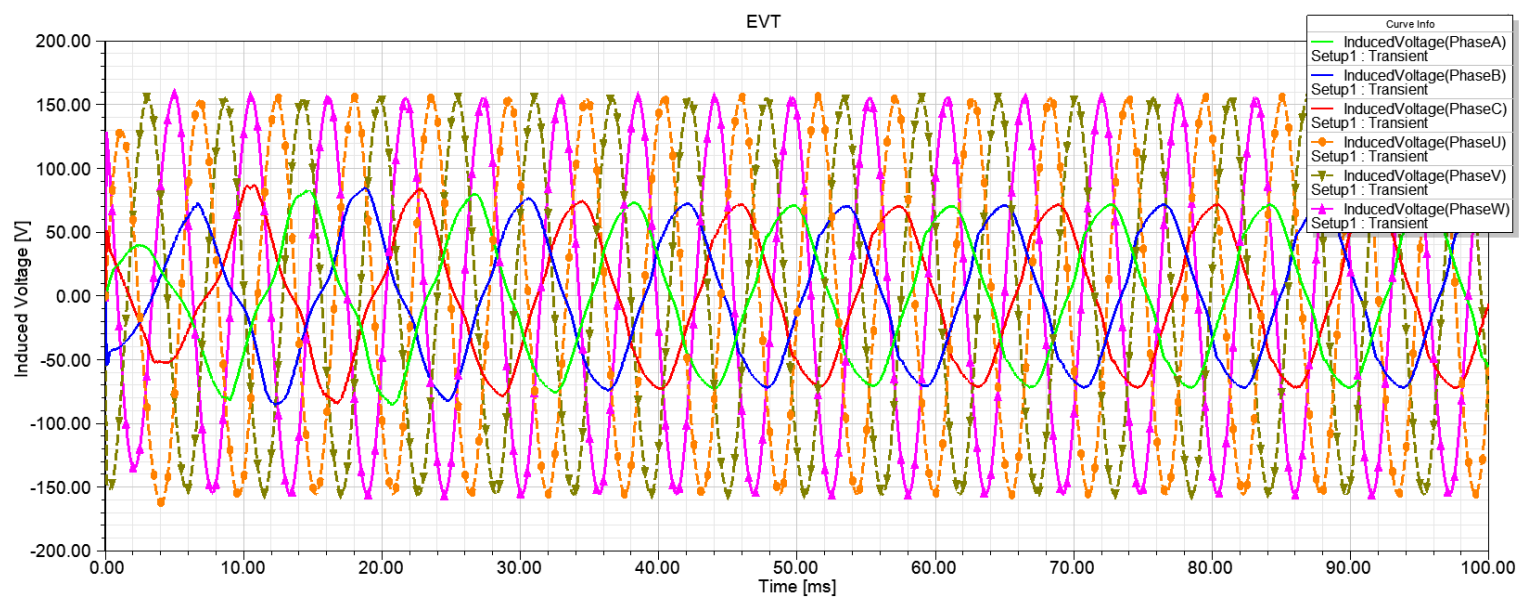

Figure 13. Induced voltage of stator windings and inner rotor windings.

After the above parameters optimization it can be seen that the induced voltages in the two windings are similar to a sinusoidal wave, which shows that the fundamental component is significant and the harmonic content is non-dominant in the air-gap. Good air-gap magnetic properties can add to the linearity of the magnetic circuit. Therefore, the parameter optimization design of the magnetic circuit is reasonable to make the magnetic field be in a low coupling state. The linearity of inductance and mutual-inductance is increased, so the output torque smoothness and the overload capacity of the EVT are improved, which makes the EVT more suitable for use in HEVs.

\subsection{Output Torque at Full-Load Operation}

Keeping the speed of EM1 and EM2 unchanged, their output torque waves are shown in Figure 14.

In Figure 14, the moving1 torque curve represents the torque of EM1, which is negative. The moving2 torque curve represents the torque of EM2, and its value is positive. This illustrates that one of the machines in operating in generating state, the other is an electric state. It also can be seen that, the torque of EM1 stablilizes at $12 \mathrm{~ms}$, which is about $16 \mathrm{Nm}$. For EM2, the value is about $76 \mathrm{Nm}$ and stablilizes at $24 \mathrm{~ms}$. The torque of EM2 decreases a little, and then increases and remains smooth. By optimizing the structural parameters and excitation current of EVT, the torque ripple of EM1 and EM2 is stable at about $5 \%$, which is generally acceptable to fulfil the stability and comfortableness demands of HEVs. 


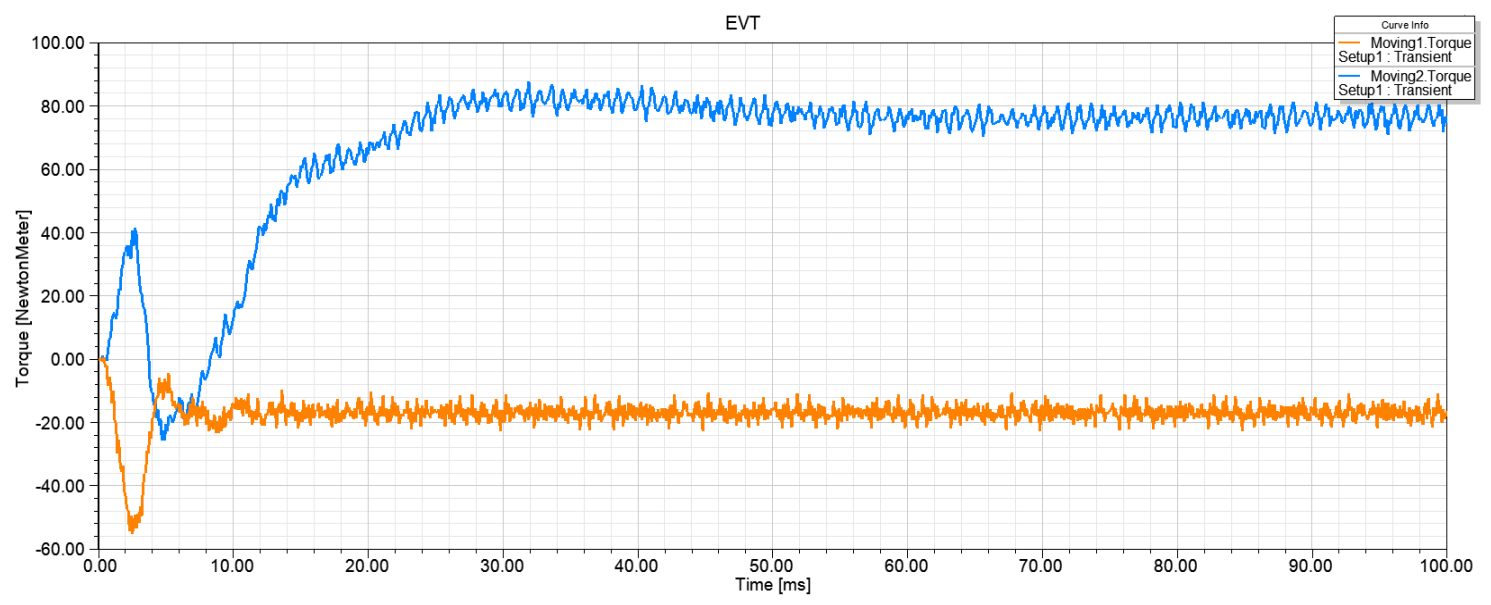

Figure 14. Output torque of EM1 and EM2.

\section{Conclusions}

An EVT with a special double rotor structure for HEVs is researched in this paper. It combines two electric machines and has few mechanical issues owing to gearless structure. However, the coupling between the inner and outer magnetic field causes torque ripple and affects the working stability of the HEVs under different load conditions. The torque mathematical model of an EVT in an $\mathrm{ABC}$ three-phase coordinate system is presented to analyze the factors that influence it. Based on the torque equations of the inner rotor and inner rotor it is seen that the magnetic field coupling causes the nonlinear variation of machine parameters, and then leads to the torque ripple. A FEM-based structural optimization design and excitation current for reducing the torque ripple is proposed. After optimization the EVT has a better magnetic field performance and a stable output torque, which verifies the reasonableness of the proposed optimal design.

Author Contributions: Conceptualization, Qiwei Xu and Shumei Cui; Data curation, Wenjuan Wang; Formal analysis, Jing Sun; Investigation, Jing Sun; Methodology, Wenjuan Wang; Project administration, Qiwei Xu; Software, Yunqi Mao; Supervision, Qiwei Xu; Validation, Yunqi Mao and Shumei Cui; Visualization, Yunqi Mao; Writing-original draft, Jing Sun; Writing-review \& editing, Jing Sun.

Funding: This work was supported by National Natural Science Foundation of China under Project No. 51507021, Chongqing Science and Technology Commission of China under Project No. cstc2013jcyjA60001, and The State Key Laboratory of Power Transmission Equipment \& System Security and New Technology in Chongqing University of China under Project No. 2007DA10512716303.

Conflicts of Interest: The authors declare no conflict of interest.

\section{References}

1. Vinot, E.; Reinbold, V.; Trigui, R. Global Optimized Design of an Electric Variable Transmission for HEVs. IEEE Trans. Veh. Technol. 2016, 65, 6794-6798. [CrossRef]

2. Xu, Q.W.; Cui, S.M.; Song, L.W.; Zhang, Q.F. Research on the power management strategy of hybrid electric vehicles based on electric variable transmissions. Energies 2014, 7, 934-960. [CrossRef]

3. Su, P.; Hua, W.; Zhang, G.; Chen, Z.; Cheng, M. Analysis and evaluation of novel rotor permanent magnet flux-switching machine for EV and HEV applications. IET Electr. Power Appl. 2017, 11, 1610-1618. [CrossRef]

4. Arunkumar, J.; Andrew, C.; Tek, T.L. Review of prospects for adoption of fuel cell electric vehicles in New Zealand. IET Electr. Syst. Trans. 2017, 7, 259-266.

5. Hoeijmakeer, M.J.; Ferreira, J.A. The electric variable transmission. IEEE Trans. Ind. Appl. 2006, 42, 1092-1100. [CrossRef]

6. Xu, Q.W.; Sun, J.; Luo, L.Y.; Cui, S.M.; Zhang, Q.F. A Study on Magnetic Decoupling of Compound-Structure Permanent-Magnet Motor for HEVs Application. Energies 2016, 9, 819. [CrossRef] 
7. Sinervo, A.; Arkkio, A. Rotor radial position control and its effect on the total efficiency of a bearingless induction motor with a cage rotor. IEEE Trans. Magn. 2014, 50, 1-9. [CrossRef]

8. Cai, H.W.; Xu, L.Y. Modeling and Control for Cage Rotor Dual Mechanical Port Electric Machine-Part I: Model Development. IEEE Trans. Energy Convers. 2015, 30, 957-965. [CrossRef]

9. Osman, C.S.; Alper, T.; Lale, T.E. Efficiency analysis in three phase squirrel cage induction motor. In Proceedings of the 2016 National Conference on Electrical, Electronics and Biomedical Engineering (ELECO), Bursa, Turkey, 1-3 December 2016; pp. 334-338.

10. Yang, Y.Y.; Schofield, N.; Emadi, A. Integrated Electromechanical Double-Rotor Compound Hybrid Transmissions for Hybrid Electric Vehicles. IEEE Trans. Veh. Technol. 2016, 65, 4687-4699. [CrossRef]

11. Kim, J.; Kim, T.; Min, B.; Hwang, S.; Kim, H. Mode Control Strategy for a Two-Mode Hybrid Electric Vehicle Using Electrically Variable Transmission (EVT) and Fixed-Gear Mode. IEEE Trans. Veh. Technol. 2011, 60, 793-803. [CrossRef]

12. Liu, Y.L.; Niu, S.X.; Ho, S.L.; Fu, W.N. A New Hybrid-Excited Electric Continuous Variable Transmission System. IEEE Trans. Magn. 2014, 50, 8104104. [CrossRef]

13. Shane, O.; Sumedha, R. A Combined High-Efficiency Region Controller to Improve Fuel Consumption of Power-Split HEVs. IEEE Trans. Veh. Technol. 2016, 65, 4597-4607.

14. Ma, Z.T.; Cui, S.M.; Li, S.P. Applying dynamic programming to HEV powertrain based on EVT. In Proceedings of the 2015 International Conference on Control, Automation and Information Sciences (ICCAIS), Changshu, China, 29-31 October 2015.

15. Liu, Y.L.; Niu, S.X.; Fu, W.N. Design of an Electrical Continuously Variable Transmission Based Wind Energy Conversion System. IEEE Trans. Ind. Electron. 2016, 63, 6745-6755. [CrossRef]

(C) 2018 by the authors. Licensee MDPI, Basel, Switzerland. This article is an open access article distributed under the terms and conditions of the Creative Commons Attribution (CC BY) license (http:/ / creativecommons.org/licenses/by/4.0/). 